\title{
STATUS OF THE LOWELL PROPER MOTION SURVEY
}

\author{
H. L. Giclas \\ Lowell Observatory
}

A proper motion survey of the northern hemisphere, utilizing first epoch plates made some thirty years ago with the 13 - inch Pluto search telescope of the Lowell Observatory, was begun in 1957. The relative proper motions of stars fainter than the eighth magnitude and with motions greater than $0 ! 26$ per year are measured directly on the calibrated grid of the projection blink comparator.

The blink examination and measurement of plates for the program has been completed in the northern hemisphere, and the comparison and referencing of the final few plates will be completed in the next month. It is then planned to combine duplicate measures from overlapping plates and print out a catalog in order of right ascension.

Data for the first twelve hours of right ascension has already been entered into the IBM 1130 for combining and ordering. It is planned that the computer will print out the catalog in consecutive page format ready for photo offset printing. About 15,000 stars have been retained and published in the Lowell Bulletins to date, and it is anticipated that there will be approximately 10,000 entries in the catalog print-out.

In addition to usual data pertinent to the proper motion, provision will be made in the programming to add as much additional useful information about a specific entry as possible. Instead of consecutively renumbering the entries in a new catalog, to further complicate identity in the literature, the original (first) G - number given the star, will identify it in the first column of the catalog. This will, also, serve to identify its chart number, which in every case, was prepared from the first time the star was identified. The charts will not be republished, but a column giving the Lowell Bulletin number in which the chart can be found will be given.

Besides other designations of previously known proper motion stars, such as Luyten, Wolf, or Ross numbers, as much other information as possible will be added. For example, for approximately 3,000 of the stars, UBV photometry has been done by several different observers. Where this data has been published, it will be given in an appropriate column together with a reference as to the source. For unpublished data, a request to include the photometry will be made, and the source acknowledged.

Several observatories are engaged in observing parallaxes of faint stars, and have included some of the G - stars on their observing programs. It is our plan to include a column in the catalog to indicate which stars have had or are in the process of having a parallax determined and by whom.

The foregoing columns, not including previous known designations, will use 84 of the 120 character lines of the IBM printer. The balance, 36 characters, will be used first for the Luyten number, followed by the Wolf, 
Ross, or BD numbers. If additional designations appear expedient, they may be continued in supplementary notes.

Those who have used the Lowell proper motions published in the Bulletins will be familiar with the Notes to Catalog, which call attention to any pertinent singularities of a star. It is planned to repeat the notes that are still useful, and to add references for as much additional information as possible.

For example, if under the photometry reference, an EG 1, 2, or 3, appears, it will denote that it appears in one of the three papers by Eggen and Greenstein, entitled, "Observations of Proper Motion Stars," appearing in the Astrophysical Journal. In all, some 346 regular program stars, and 259 of the GD - stars have been spectrally classified by them. Sandage, in his search for subdwarfs, has, with Kowal, done the UBV photometry of nearly 2,000 stars of color class +2 or bluer and brighter than 14th magnitude. Over 300 subdwarfs have been found, and radial velocities have been determined for 80 of these.

The completed catalog will be stored on an 1130 disk, and various programs have already been written to utilize the data in many ways. One example is a program to list stars between any given magnitude, color, motion or P.A. range in different areas of the sky that are moving toward a given convergent. The observed position angle is compared directly to the computed. The differences are then plotted and examined for an excess number of stars moving toward a convergent. In the case of the Hyades, an excess number moving towards the conyergent have been found and these have been listed and reported previously. Recently, the available data has been searched for possible additional members of the Ursa Major group, but without success. Since there may be candidates with proper motions smaller than the Lowell limit, all stars with motions less than 0!'27 per year listed in the LTT catalog, and moving within ten degrees of the Ursa Major convergent were investigated for possible membership, but again no excess number of stars were found moving toward this convergent. Other programs will plot the number of stars moving in each ten degree interval of position angle between any limits of magnitude, color, or amount of motion. The solar motion derived with reference to stars of different magnitude limits and colprs has been reported on previously at the Charlottesville conference.

Upon completion of the northern hemisphere print out, the survey will continue in the southern. Some supplementry things we want to do are to re-examine the first fifty plate regions for white dwarf suspects and extremely red ones. On many of these earliest regions, yellow instead of red plates were used to determine colors, and we have found the increased spectral range facilitates greatly identification of both white and red dwarfs. We have not, heretofore, looked particularly for excessively red stars, but Greenstein's recent attempt to resolve the discrepency in the number of white as compared to red degenerate stars has challenged observers to look 
for them. Only about one-fifth as many red degenerates are known as compared to white. Greenstein has suggested that this may be because they pass quickly through the red stage as they grow fainter. We will begin marking the faint very red stars with small proper motion, as well as, the blue.

The illumination of the night sky from the city of Flagstaff has increased so much in the last few years that it will be necessary to move the large field telescope used for the program to a dark sky site, twelve miles southeast of its present location. In continuing the program into the southern hemisphere, it will then be possible to match first epoch plates around the south galactic pole made in 1934, and to do a proper motion study in that area in depth.

(1) Eggen, O.J. and Greenstein, J. L.

1965a, Ap. J. , 141, 83 (Paper I) ibid. , 142, 925 (Paper II) 1967, ibid. , 150, 927 (PaperIII)

(2) Sandage A. R. 1964a, Ap. J., 139, 442 (Paper I)

(3) Giclas, H. L. 1969, ibid. , 15. 1115 (Paper II) 1969, Paper I-2, Low-Luminosity Stars, Kumar, Gordon and Breach Sci. Pubs., London

\section{DISCUSSION}

Luyten: In connection with the yellow and reddish white dwarfs, I am now working - in anticipation of St. Andrews Conference - on a complete catalogue of all the degenerate stars - all the possibles and probables and what not that have come out of the two surveys. As of now I have about 3,000 entries in the catalogue, and a substantial number are of color class $\mathrm{g}$ or $\mathrm{k}$. There it is very difficult to be sure that the color of an ordinary $M$ dwarf has not been underestimated and I am sure the casualties will be very high. As to degenerates of color $m$ - I now have five possible candidates, all of them components of doubles. Normally, in a double, in which one component is degenerate and the other is a normal red main sequence star, the main sequence star is, bolometrically nearly always the brighter. But when the redder star is bolometrically the fainter, as in a probable double white dwarf, then I think we have a chance to find red degenerates. 